\title{
New ways with interatomic forces
}

\author{
Attempts to calculate the properties of real materials from those of their constituents have never been \\ outstandingly successful. New, but still empirical, techniques may help.
}

SinCE the year dot, or thereabouts, all kinds of people have been calculating the properties of matter of various kinds by making assumptions they know to be inadequate about the forces between constituent particles. The process continues, and discontent with the assumptions is, if anything, made more explicit by the availability of computers and the associated computer codes that will allow the treatment of systems large enough to be considered "realistic". But supply tends to grow to meet demand, and the invention of novel schemes for representing interatomic forces is now also flourishing. One such is a way of representing long-range interatomic forces developed by a group based at the University of Trieste (F.Ercolessi, E. Tosatti and M. Parrinello) and applied to the structure of the surface of gold crystals (Phys. Rev. Lett. 57, 719; 1986).

The problem of calculating ab initio the properties of a material is indeed intractable. Even that model of the ideal gas in which the atoms are perfectly elastic solid spheres is useful only for as long as the spheres are not so large, relative to their container (again assumed perfectly elastic) that geometrical effects make them clump together in some sense. More realistically, the forces between pairs of atoms in, say, a monatomic gas are better represented by a distance-dependent mutual potential energy leading to forces that are repulsive at short distances (reflecting the solidity of atoms) which may be offset by attractive forces at greater distances. Van der Waals is the name to conjure with; physically, what happens is that random intra-atomic fluctuations giving individual atoms an electrical dipole moment will tend to synchronize with the instantaneous dipole moments of interacting atoms, dragging the two together, which is why gases whose constituents are either polar or highly polarizable molecules depart most conspicuously from the perfect gas laws.

Mischievously, real substances are more cussed than these simple examples allow. Even in gases, three and multibody forces are bound to be relevant, and properties may depend not only on the positions of atoms or molecules instantaneously, but on their velocities as well. A further complication, especially when the average inter-particle distance is small, is that there may be literally no limit to the distance over which inter-particle forces exert themselves because of cooperative effects in which the miscroscopic interactions have the effects of enforcing longrange order, when a material behaves as if the range of the physical force between a particle and the environment of which it is a part is literally infinite.

So what happens in, for example, a metal, which may be taken, for the sake of argument, to be a lattice of positively charged ions embedded in a sea of negative electricity? Simple calculations based on the assumption that the ions interact with each other by some defined interionic potential energy will not wash, because the electron sea is of necessity a long-range system whose collective properties and, in partucular, energy may not be simply expressed by the distance between the members of an arbitrarily chosen pair of atoms.

Ideally, people wishing to calculate the binding energy of a metal should proceed differently: first, make an assumption about the geometry of the ion lattice, then calculate the allowable quantum states of electrons, finally allow that the electron distribution will seek a lower level of energy in which ionic charges are more completely shielded from each other (which is where the Thomas-Fermi and other selfconsistent field calculations come into their own). The results are reasonable enough, although not especially persuasive, for one thing because nobody is confident of being able to adapt the selfconsistent calculations to the real case in which the ions of the ion-lattice are in motion, as they must be.

That is why there is such ample room for more empirical ways of tackling the problem by the invention of novel ways of describing the forces between ions in, say, a metallic crystal. The obvious difficulty is that the bulk properties of metals do not provide a sufficiently stringent test of possible refinements of assumed interatomic force laws, all of which must have certain general properties in common. But distortions at the surfaces of metallic crystals to the regular distribution of ions in the bulk may be an opportunity for verification, which is where Ercolessi and his colleagues begin, with the surface structure of metallic gold.

Like many other solid lattices, but more markedly, the surface of a metallic gold crystal is not a simple projection onto a crystallographic surface of the structure of the crystal lattice in bulk. Instead, the atoms of this structure, whose unit cell is face-centred cubic, appear on simple crystallographic surfaces - such as $(001)$ - to be arranged in stripes, five rows of atoms wide within which atoms are arranged on a triangular rather than a square pattern. This has been shown experimentally by, for example, low-angle electron diffraction.

Ercolessi et al. apply to this structure a force-law they have developed in other connections, one that allows not only for pair-wise forces between atoms (including other than pairs of nearest neighbours) but for a generalized glue-like force which has the effect of making it energetically adventageous, in a system such as a metallic crystal, that the coordination number should be increased. Their two-body force is conventional enough, implying repulsion at short distances, a minimum energy at about $0.27 \mathrm{~nm}$ and attractive forces at greater distances. The glue force is harder to visualize, but represents a kind of tradeoff between high coordination number and proximity between pairs of atoms.

The outcome is remarkably suggestive even though it leaves much to be desired. By a suitable choice of the empirical constants in the force laws, five-row stripes of atoms can indeed be made to appear on the simple surfaces of gold crystals. The obvious snag is that there seems very little hope of being able to calculate either the pair-wise forces or those that represent the glue from what might be called first principles. Even so, especially because of the importance of, for example, the surface structure of semiconducting materials such as silicon, there is every prospect that this new technique of calculation will quickly become fashionable. It may even become a fashion that will last.

The situation in the calculation of the properties of metals, in other words, may soon not be very different from that in the calculation of the properties of gases half a century ago. Then it was that people were aware of the complexity of the pair-wise interatomic force as well as of the need to pay some attention to three and multibody forces. The force-laws were for the most part empirical, but it is remarkable how useful they proved to be. Nobody will weep if there is a period, perhaps a long one, during which the calculation of the properties of metals is as much a matter of finding, through trial and error, which methods work and which do not.

John Maddox 